\title{
IMPEDANCE IMAGING FOR HYBRID BIOSENSOR APPLICATIONS
}

\author{
D. A. Borkholder ${ }^{1}$, N. I. Maluf ${ }^{1,2}$, and G. T. A. Kovacs ${ }^{1}$ \\ 'Stanford University \\ Center for Integrated Systems Room 202X \\ Stanford, CA 94305-4075 \\ ${ }^{2}$ Lucas NovaSensor
1055 Mission Court
Fremont, CA 94539
}

\begin{abstract}
The impedance characteristics of individual cell / electrode systems are used to monitor cellular viability, position, adhesion, and response to external stimuli in hybrid biosensor applications. A planar microelectrode array consisting of 36 platinized iridium electrodes $(10 \mu \mathrm{m}$ diameter) is used as a substrate for the culture of mammalian cells. Electrode impedance is monitored across the array as different environmental factors are changed. Maps of electrode impedance have been shown to correlate directly to cell positioning over an electrode and general cellular viability. Exposure to a well known voltage-gated $\mathrm{Na}^{+}$channel blocker (tetrodotoxin) provided significant cellular response as compared to control electrodes without cells. The effective use of small electrodes $(10 \mu \mathrm{m}$ diameter $)$ to study single cell/ electrode interactions has been demonstrated.
\end{abstract}

\section{INTRODUCTION}

Much of biosensor development aims to utilize the highly selective and sensitive nature of biological compounds to detect a particular analyte. However, there is interest in developing sensors with sensitivity to a wide variety of physical and chemical stimuli. Whole cell based biosensors offer such diversity while maintaining high sensitivity. Utilizing planar microelectrode technology, it is possible to monitor the electrical activity of anchorage dependent cultured cells [1], as well as the impedance characteristics of the electrode/cell system [2-7]. It has been suggested that it is possible to detect the attachment, spreading, movement, and division of cells plated on a relatively large $(250 \mu \mathrm{m}$ diameter) single electrode with the impedance characteristics dependent on the average response of populations of cells [5].

The reaction of anchorage dependent mammalian cells to different environmental factors may be monitored by impedance measurements of the electrode / cell interface. The resulting impedance is dependent on many variables including degree of occlusion of the electrode surface, relative adhesion of the cell to the electrode, and cell membrane ion channel conductance. Thus, it is possible to monitor cell positioning over an electrode, cellular viability and mobility, and the introduction / removal of agents which modulate ion channel conductance (or any of the other factors mentioned above). The technique is potentially usable with a far broader range of cell types than other techniques that require cell generated action potentials for signal transduction.

NG108-15 (neuroblastoma $x$ glioma) hybrid cells [8] have been utilized extensively for many types of morphological and biochemical studies. They express at least four major families of voltage-sensitive channels, including voltage-gated sodium channels, that respond to a variety of ion channel blockers [9]. Tetrodotoxin (TTX) is a highly specific blocker of voltage gated $\mathrm{Na}^{+}$channels. TTX binds to the outer mouth of the channel through which sodium ions move, thereby physically blocking ionic current through the channel [10]. Thus it is a useful substance for testing the applicability of impedance imaging for hybrid biosensors.

Chemical and biological warfare (CBW) agents pose a difficult detection problem due to the broad (and ever expanding) spectrum of materials that may be used. Thus, there is a need for detection systems that exhibit high sensitivity while maintaining diverse detection capabilities. Whole cell based biosensors provide this critical combination. The use of impedance imaging allows for a large number of different cell types to be cultured, thereby allowing for a tunable sensor response. This technology promises to be a complementary component to existing CBW detection systems. Also of great interest is the use of such techniques for pharmaceutical screening, both for intended agent effects and for toxicological studies. A variety of basic biological studies could also potentially benefit from this approach. In the latter two cases, this type of assay could reduce the number of animal-based tests required.

\section{MATERIALS AND METHODS}

A $6 \times 6$ array of $10 \mu \mathrm{m}$ diameter planar microelectrodes ( $100 \mu \mathrm{m}$ pitch) has been fabricated for cultured cell studies. A $500 \mathrm{~nm}$ thermal oxide provides electrical isolation from the underlying silicon substrate. Iridium electrodes and gold interconnects and bondpads are deposited utilizing lift-off techniques, followed by deposition of a $1 \mu \mathrm{m}$ low stress PECVD silicon nitride passivation layer. Vias are etched through the nitride to define the electrodes and bondpad openings. The $9 \times 9 \mathrm{~mm}$ chips are packaged in a standard 40-pin ceramic dual in-line package. The bondwires are encapsulated (for mechanical and electrical robustness) in a nontoxic low stress epoxy.

An impedance imaging system (Figure 1) has been developed to monitor the impedance of cell / electrode interfaces across the electrode array. A quadrature synthesizer is used to generate both sine and cosine signals of programmed frequency and amplitude. The 10V P-P sinewave signal is attenuated as needed (from $0 \mathrm{~dB}$ to $-80 \mathrm{~dB}$ ) and connected to an individual electrode via an analog multiplexer. The resulting current is monitored by a transimpedance stage which holds the large reference electrode at virtual ground. Automatic gain control $(0 \mathrm{~dB}$ to $100 \mathrm{~dB})$ is used to amplify the voltage output of the transimpedance stage. This amplified signal is multiplied in quadrature by the source signal and low pass filtered (fourth order Butterworth at $10 \mathrm{~Hz}$ ) to provide the real and imaginary components of the measurement. Quadrature multiplication (a common lock-in amplifier technique) allows for signal detection (at the excitation frequency) with high noise immunity. Known value resistors are used to calibrate the system for electrode impedance measurements. 


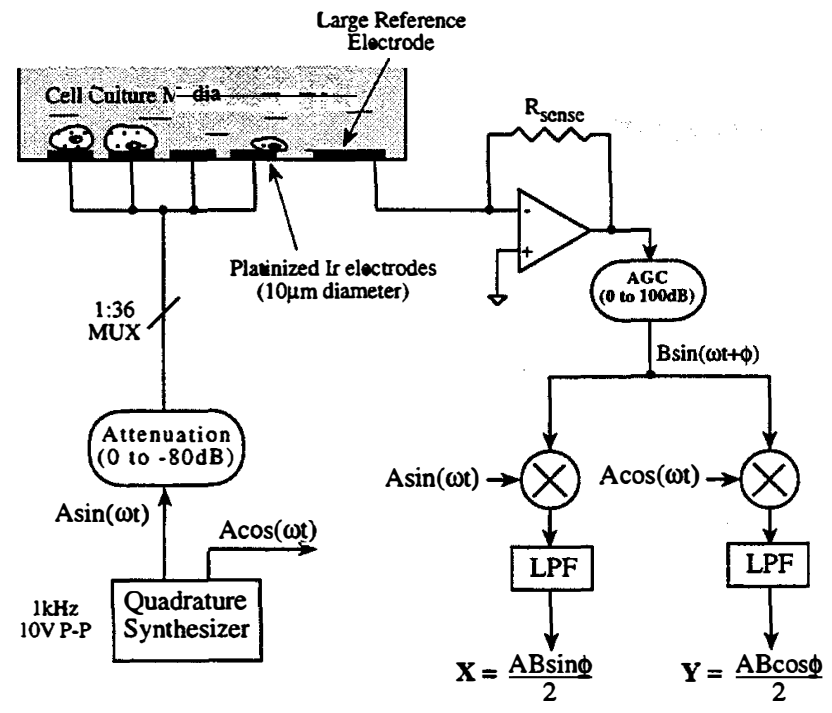

Figure 1. Computer controlled system used for monitoring the impedance of cell / electrode interfaces. An AC voltage is used to drive one small electrode at a time; the resulting current is converted to a voltage, amplified, and homodyned to yield the real and imaginary parts of the electrode impedance. The small electrode dominates the measured impedance.

The temperature of the substrate is regulated by an analog feedback loop using a cold-junction compensated thermocouple and a solid state Peltier device [11]. Achieved regulation at $37^{\circ} \mathrm{C}$ is better than $\pm 0.3^{\circ} \mathrm{C}$.

NG108-15 cells are cultured on the electrode arrays in serum containing media using standard cell culture protocols [9]. The chips are placed in a $\mathrm{CO}_{2}$ incubator $\left(37^{\circ} \mathrm{C}, 10 \% \mathrm{CO}_{2}\right)$ for 24 to 48 hours (long enough to ensure adequate adhesion to the electrodes). For impedance measurements, the chips are removed from the incubator and placed into the measurement system where the temperature is maintained at $37^{\circ} \mathrm{C}$. While atmospheric $\mathrm{CO}_{2}$ concentration is not controlled, buffers in the culture medium adequately maintain the $\mathrm{pH}$ for the duration of the impedance measurements.

A $1 \mathrm{kHz}, 100 \mathrm{mV}$ P-P sinusoidal wave (small enough to ensure linear electrode characteristics [12]) is applied to a single electrode in the array. The resulting current flowing through a large reference electrode into a virtual ground is used to calculate the effective impedance of the cell/electrode interface combination. Electrode impedance is monitored across the array at approximately three minute intervals. The culture media is exchanged for new media with the same formulation in order to "normalize" the solution $\mathrm{pH}$ for subsequent addition of toxins prepared in the same solution (Significant $\mathrm{pH}$ changes can occur during the 24 to 48 hour culture period due to cell metabolic processes; this effect must be normalized out to perform meaningful cellular response studies). Culture media containing the substance to be tested is exchanged for the existing media and the resulting impedance changes are monitored over time. After a leveling of impedance response, the test solution is rinsed twice and replenished with new cell culture media. Again, the resulting impedance is monitored. Finally, the cells are rinsed an additional time to ensure full removal of any remaining test solution. It is important to note that this may not guarantee full reversal of a toxin's effects.

Impedance measurements across the array allow for determination of cell position and coupling to electrodes when the measurements are normalized to data taken prior to cell plating. Where detection of environmental factors or other external stimuli is desired, the impedances are normalized to the first measurement of a series.

This entire sequence was repeated for an identical control chip which underwent all treatments and culture protocols without the addition of cells. This data ensures impedance changes observed with changing environmental factors are due to biological effects rather than changes in adsorbed substances on the electrodes themselves.

\section{RESULTS}

System performance and variability were evaluated by measuring a $1 \mathrm{M} \Omega, 1 \%$ metal film resistor in place of the electrode array (measured electrode impedances at $1 \mathrm{kHz}$ typically range from $10 \mathrm{k} \Omega$ to $2 \mathrm{M} \Omega$ depending on cell coupling). With twenty trials, maximum single channel variations of $0.11 \%$ and channel-to-channel variations of $0.12 \%$ in impedance magnitude were observed. This is indicative of the anticipated measurement noise due to the electronics.

Impedance measurements normalized to the bare chip impedances were used to directly correlate cell placement over electrodes. Figure 2 presents impedance data for healthy cells growing on the electrode array. Regions with cells positioned directly over an electrode (highlighted with boxes in the optical image) correspond to regions of high impedance as can be seen from the 2-D impedance map in Figure $2 b$. In applications where action potential signals are to be measured from the cultured cells, tight cell / electrode coupling is desirable for improved signal-to-noise ratio (SNR) [13]. Thus, impedance imaging may be used as a complementary technology to determine which electrodes are covered by tightly coupled cells and should be monitored for optimal SNR.

Lysing (causing cellular death by generation of different osmolality inside and outside of the cell, resulting in rupture of the cellular membrane) the cells by perfusion with a hyperosmolar solution resulted in the data presented in Figure 3. A clear decrease in measured impedance across the array is evidenced, corresponding to lifting of cells and partial removal of adsorbed biological substances from the electrodes. While this is a drastic demonstration of cellular viability assessment, it also illustrates the sensitivity of the impedance measurements to adsorbed materials and the necessity of controls.

To examine the more subtle effects of ion channel blockage, single electrode impedances were monitored over time as such toxins were added. Optical images of several representative electrodes are shown in Figures $4 a$ and $5 a$ at the beginning of the experiment. The impedance magnitude of the indicated electrodes are tracked in Figures $4 \mathrm{~b}$ and $5 \mathrm{~b}$. Small effects were observed as the culture media was replaced with fresh medium, probably due to $\mathrm{pH}$ differences between the two solutions. Cellular metabolism results in acidification of the culture media and becomes more pronounced over time as buffering action is depleted. Thus, perfusion with fresh solution 

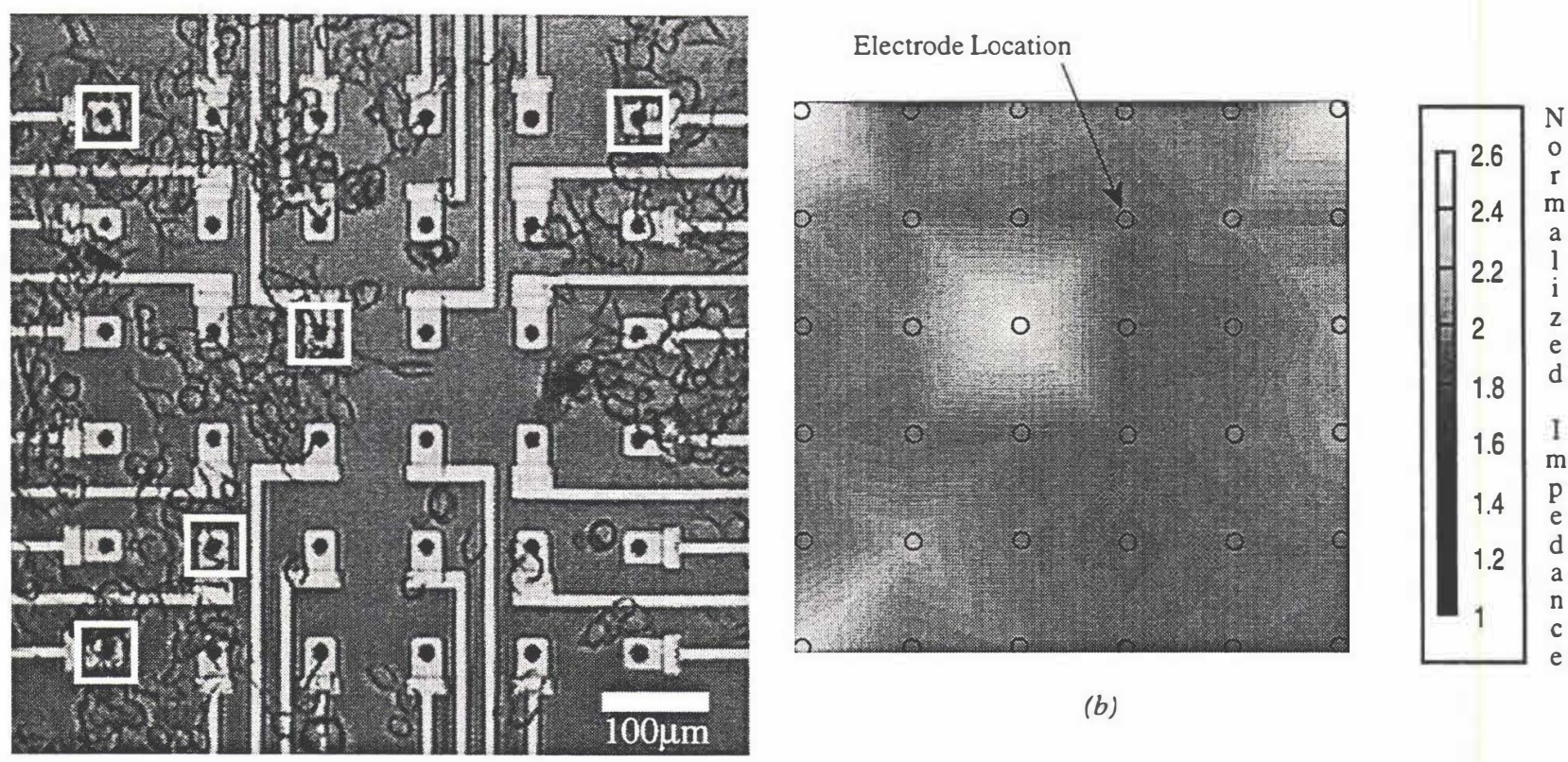

(a)

Figure 2. Cultured NG108-15 hybrid cells. a). Optical photograph of cells growing on the electrode array. Boxes indicate electrodes with apparent tight cell / electrode coupling as seen by the impedance map in (b). b). 2-D impedance magnitude map normalized to the bare chip impedance. Interpolation was used to form contours between discrete data points.
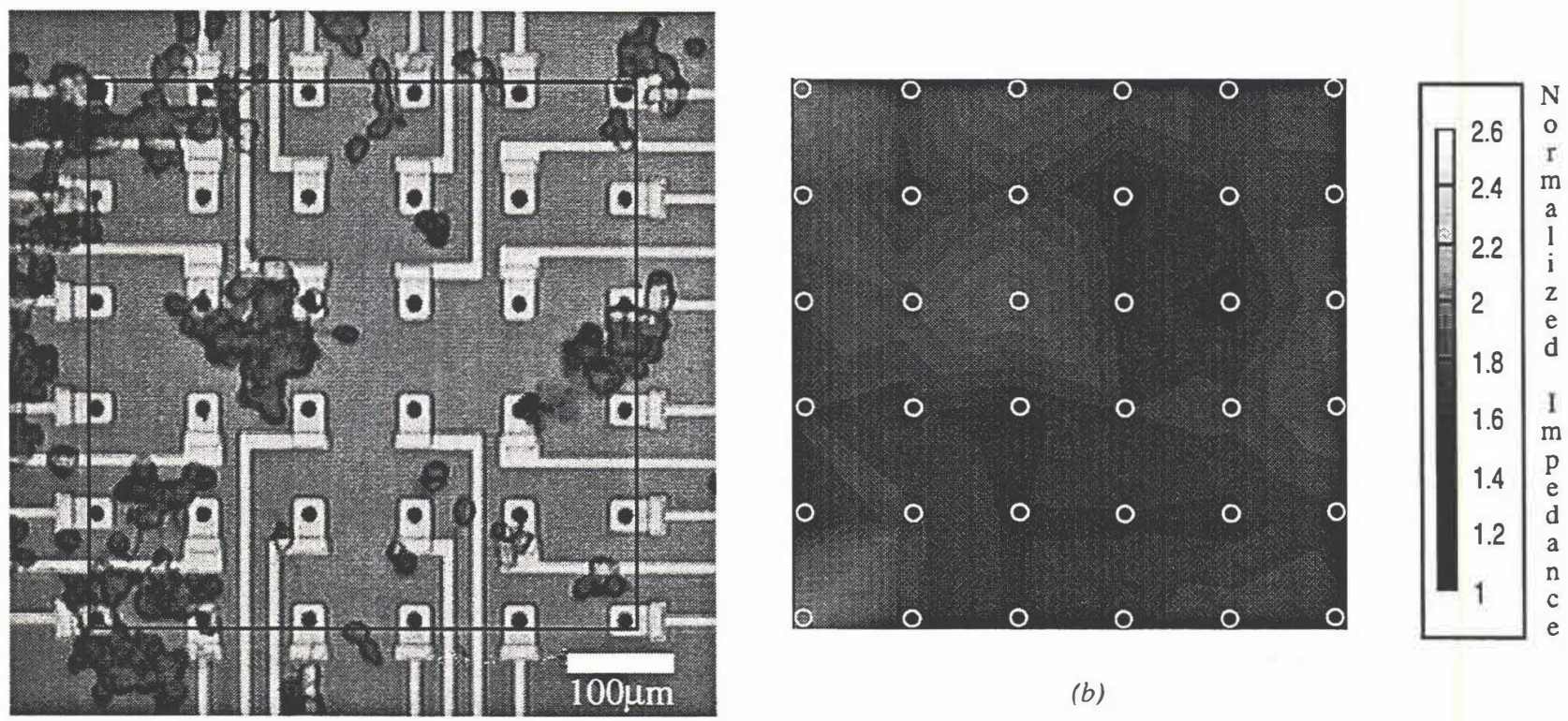

(a)

Figure 3. NG108-15 cells following lysing in a hyperosmolar solution for five minutes. a). Optical photograph of cells on the array. b). 2-D impedance magnitude map normalized to the bare chip impedance. Note overall decrease in impedance due to lifting of cells and partial removal of adsorbed biological substances.

results in increased $\mathrm{pH}$ which is evidenced by the change in measured impedance.

The addition of the sodium channel blocker tetrodotoxin (TTX) resulted in qualitatively different responses from different electrode / cell combinations. The most pronounced effects may be seen with traces A (Figure $4 \mathrm{~b}$ ) and $C$ (Figure $5 \mathrm{~b}$ ). With the addition of TTX, the cell clump over electrode A (Figure 4a) responded with a drastic increase $(180 \%)$ in measured impedance magnitude, most likely corresponding to blockage of voltage-gated $\mathrm{Na}^{+}$channels. As these channels are blocked, the resulting impedance is expected to increase due to decreased resistive conductance through them. This particular clumped cell response did not recover upon removal of the TTX solution.

The optical image of Figure 4 a shows complete electrode coverage by a single cell on electrode B. The response in Figure $4 \mathrm{~b}$ follows that described by trace $\mathrm{A}$ except that the cell recovers from the TTX exposure. It is important to note that statistically significant data has not been gathered on variability of cell 


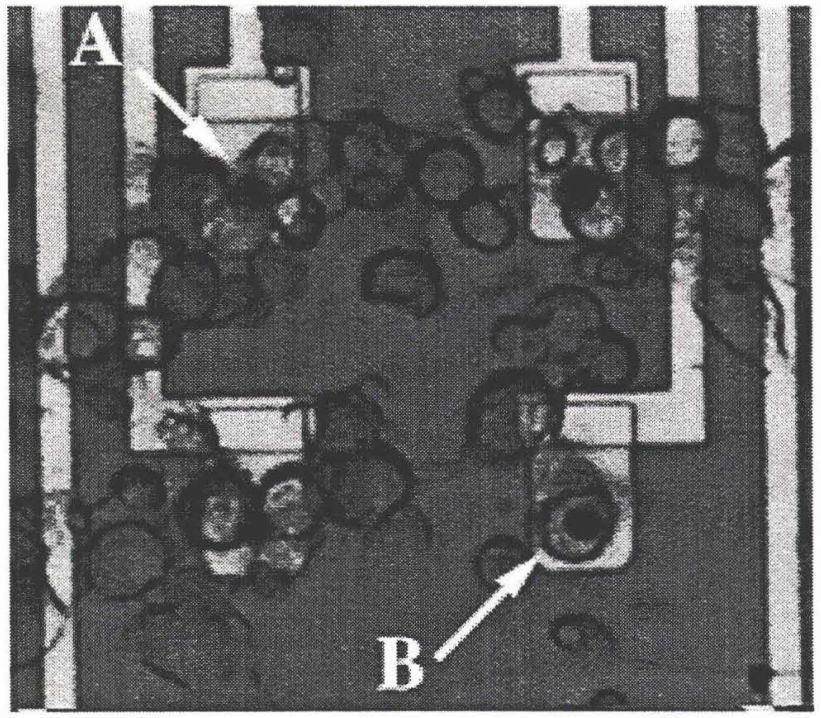

(a)

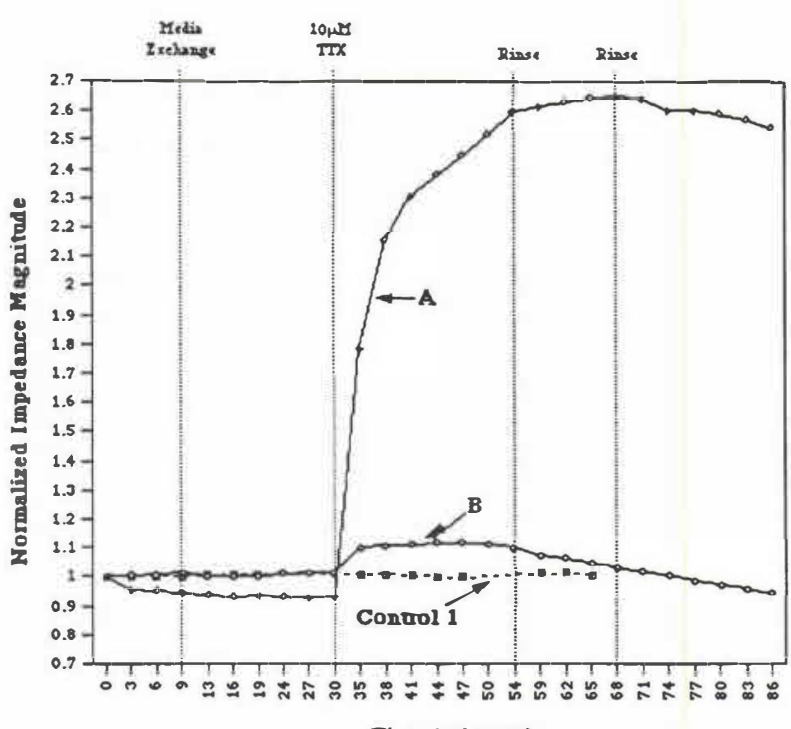

Time (minutes)

(b)

Figure 4. (a). Optical image of cells plated over electrodes $A$ and B. Note multiple cells positioned over electrode $A$ while a single cell completely covers electrode $B$. (b) Normalized impedance magnitude for electrodes $A, B$ and Control 1 . Note near constant response for Control 1 (representative response of control electrodes without cells), while both $A$ and $B$ respond with an increase in impedance magnitude with the introduction of TTX. Impedance magnitudes were on the order of 10-50k $\Omega$.

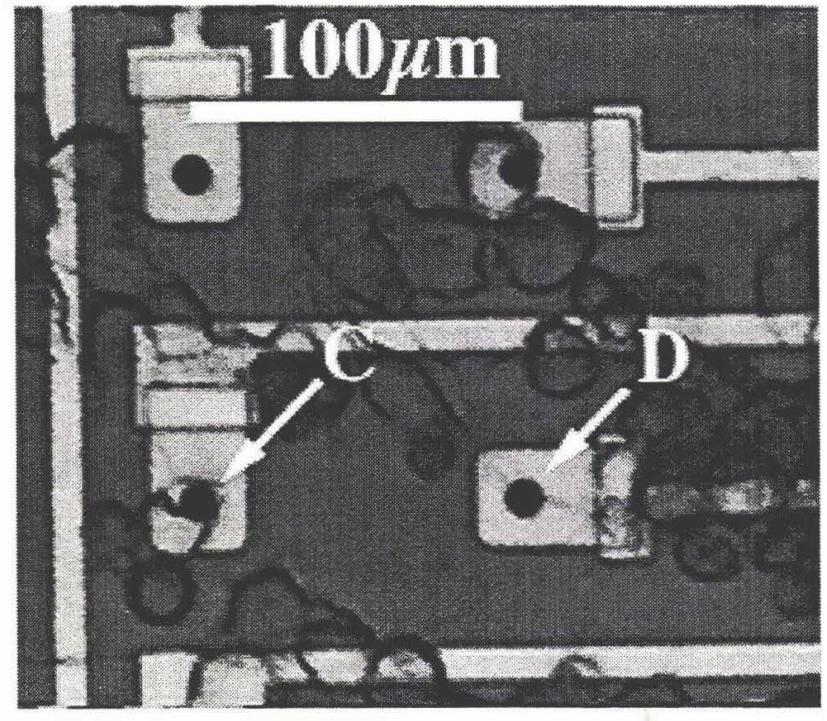

(a)

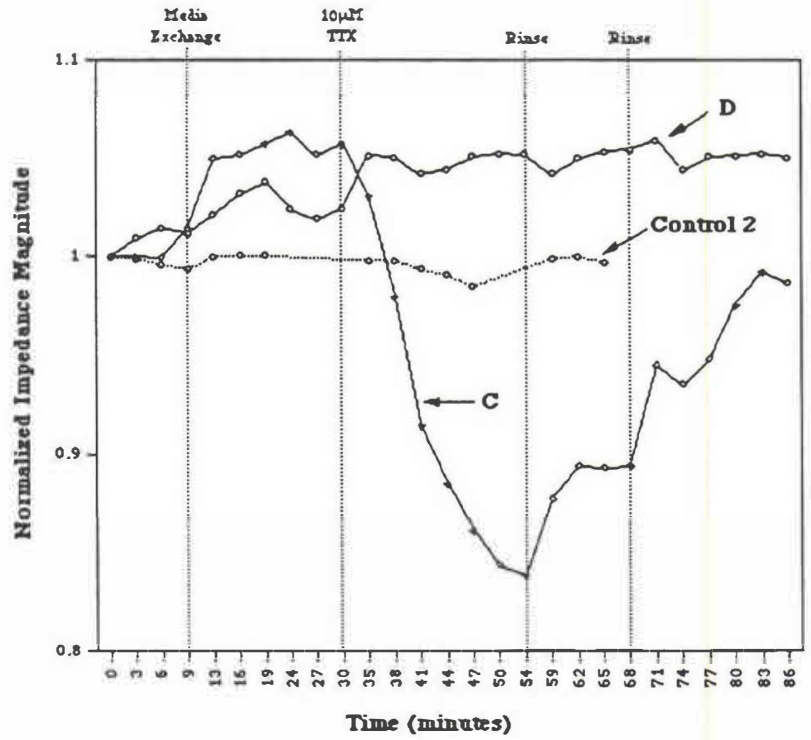

(b)

Figure 5. (a). Optical image of cells plated over electrodes $C$ and $D$. Note single cell partially covering electrode $C$ and no cells covering electrode $D$. (b). Normalized impedance magnitude for electrodes $C, D$ and Control 2. Note near constant response for Control 2 (representative response of control electrodes without cells), minimal response for $D$ (no cell over electrode) and a decrease in impedance magnitude for electrode $C$ corresponding to the introduction of TTX. Rinsing the TTX away results in a net rise in electrode $C$ impedance magnitude. Impedance magnitudes were on the order of $50 \mathrm{k} \Omega$ to $200 \mathrm{k} \Omega$

sensitivity to TTX and its reversible effects as related to impedance measurements of this type.

Electrode $\mathrm{C}$ exhibited a qualitatively different response to the TTX addition, with a resulting impedance magnitude decrease of $20 \%$. Removal of the TTX solution resulted in an impedance magnitude rise to a constant level $10 \%$ below the pre-TTX value. A second rinse resulted in increased impedance to within $1.6 \%$ of the pre-TTX value. The mechanism for this behavior is not yet understood. Note minimal changes on an adjacent electrode without coupled cells (electrode D). 


\section{DISCUSSION AND SUMMARY}

A system has been presented that monitors cellular viability and position by impedance imaging, demonstrating potential use in a variety of sensors. Maps of electrode impedance have been shown to correlate directly to cell positioning over an electrode array. While a thorough investigation of the mechanisms affecting cellular response to a well known voltage-gated $\mathrm{Na}^{+}$channel blocker (TTX) has not been undertaken to date, the results presented indicate significant cellular response as compared to control electrodes without cells. The effective use of electrodes small enough (10 $\mu \mathrm{m}$ diameter) to study single cell / electrode coupling has been demonstrated. Investigations of this and other agents along with tight control (and purposeful variation) of environmental factors utilizing this system's full capabilities (frequency variation over five decades and signal source amplitude variation) should allow for development of detailed models of cell / electrode coupling and the mechanisms affecting cell membrane impedance.

By utilizing different cell types, it is possible to tune the sensitivity of the sensor to a specific application. Nonelectrically active cell types such as hepatic cells could be used for toxin detection. For sensors utilizing electrically active cells, impedance imaging may be used to determine which electrodes are covered by cells (and therefore should be monitored), and as a secondary monitor of cellular viability. Possible application areas include: neurotoxin detection, cell cytotoxicity testing, pharmaceutical screening, and environmental monitoring.

\section{ACKNOWLEDGMENTS}

This work was funded through a combination of G. Kovacs' NSF NYI Award (No. ECS-9358289) and Terman Fellowship (courtesy of Mr. D. Packard and Mr. W. Hewlett) and a grant from the Office of Naval Research (No. N00014-93-1-0759). D. Borkholder is supported by an Air Force Fellowship.

\section{REFERENCES}

[1] G.W. Gross, "Simultaneous single unit recordings in vitro with photoetched laser deinsulated gold multimicroelectrode surface", IEEE Trans. Biomed. Eng. $26,273-279$ (1979)

[2] M. Kowolenko, C.R. Keese, D.A. Lawrence, I. Giaever, "Measurement of macrophage adherence and spreading with weak electric fields", Journal of Immunological Methods, Vol. 127, 71-77 (1990).

[3] C.R. Keese, I. Giaever, "A whole cell biosensor based on cell-substrate interactions", Annual International conference of the IEEE Engineering in Medicine and Biology Society, Vol. 12, No. 2, 1990.

[4] P.M. Ghosh, C.R. Keese, I. Giaever, "Monitoring electropermeabilization in the plasma membrane of adherent mammalian cells", Biophys. Journal, Vol. 64, May 1993, 1602-1609.

[5] C.R. Keese, I. Giaever, "A biosensor that monitors cell morphology with electrical fields", IEEE Engineering in Medicine and Biology, June/July 1994, 402-408.
[6] I. Giaever, C.R. Keese, "Use of electric fields to monitor the dynamical aspect of cell behavior in tissue culture", IEEE Transactions on Biomedical Engineering, Vol. BME-33, No. 2, Feb. 1986.

[7] P. Connolly, G.R. Moores, W. Monaghan, J. Shen, "Microelectronic and nanoelectronic interfacing techniques for biological systems", Sensors and Actuators B, 6 (1992) 113-121.

[8] C.N. Christian, et al., "Synapse formation between two clonal cell lines", Science, 196: pp.995-998 (1977).

[9] V.C. Kowtha, et al., "Comparative electrophysiological properties of NG108-15 cells in serum-containing and serum-free media", Neurosci. Lett., 164: pp.129-133 (1993).

[10] B. Hill, Prog. Biophys. Mol. Biol. 21: 1-32 (1970).

[11] N.I. Maluf, et al., "A thermal signal generator probe for the study of neural thermal transduction", IEEE Transactions on Blomedical Engineering (July 1994) vol.41, no.7, pp. 649-55.

[12] G.T.A. Kovacs, "Microelectrode models for neural interfaces", pp.121-165, in D. A. Stenger, T. M. McKenna (Eds) (1994), "Enabling Technologies for Cultured Neural Networks", Academic Press, San Diego, CA.

[13] Nicholls, Martin, and Wallace, "From Neuron to Brain", (1992) pp. 32-34, Sinauer Associates, Sunderland, MA . 